\title{
SPACES OF AFFINE CONTINUOUS FUNCTIONS ON SIMPLEXES $\left({ }^{1}\right)$
}

\author{
BY \\ ALDO J. LAZAR
}

1. Introduction. Let $C$ be a convex subset of a linear topological space and $E$ another linear topological space. Suppose that $T$ is a map from $C$ to $2^{E}$, the family of all nonempty subsets of $E$. An affine continuous function from $C$ to $E$ is called an affine continuous selection for $T$ if $f(c) \in T(c)$ for every $c \in C$. The first aim of this paper is to find sufficient conditions for the existence of an affine continuous selection for $T$ when $C$ is a compact convex subset of a locally convex linear topological space. Two restrictions imposed on $T$ are quite natural in this connection. We confine ourselves only to affine maps $T$, that is, maps for which $T(c)$ is a nonvoid convex subset of $E$ for every $c \in C$ and

$$
\lambda T\left(c_{1}\right)+(1-\lambda) T\left(c_{2}\right) \subset T\left(\lambda c_{1}+(1-\lambda) c_{2}\right)
$$

whenever $0<\lambda<1$ and $c_{1}, c_{2} \in C$. Besides, we require from $T$ to be a lower semicontinuous map i.e., for any open set $U \subset E,\{c \in C: T(c) \cap U \neq \varnothing\}$ is a relatively open subset of $C$. The existence of continuous selections for lower semicontinuous maps defined on topological spaces was intensively studied by Michael in a series of papers from which [17] and [18] are the closest to our subject. Michael showed that if a set-valued map has in a certain sense enough continuous selections it must be lower semicontinuous. We use here some of the methods and the patterns developed by Michael.

We prove that the problem of finding an affine continuous selection has always a solution when $E$ is a Fréchet space if and only if the domain of $T$ is a simplex (Theorem 3.1, Corollary 3.3). A compact convex subset $K$ of a locally convex linear topological space $F$ is called a simplex if the cone $W$ with the origin as vertex and having $K$ as its base induces a lattice ordering in the subspace $C-C$. Of course, we tacitly assumed that $K$ is situated in a hyperplane of $F$ not passing through the origin but it is easily seen that this is not an essential restriction. This definition expresses an intrinsic property of $K$ and it is equivalent with the uniqueness of integral representations of the points of $K$ by maximal probability measures on $K$. The fundamental properties of simplexes may be found in [5] or [20].

Received by the editors December 6, 1966.

(1) This paper is part of the author's Ph.D. thesis prepared at the Hebrew University of Jerusalem under the supervision of Professor A. Dvoretzky and Dr. J. Lindenstrauss. The author wishes to express his acknowledgment to both for their helpful guidance and kind encouragement. 
The selection theorem we prove helps us to investigate those Banach spaces which admit a representation as spaces of affine continuous functions on simplexes. Semadeni [21] proved that a compact convex set is a simplex if and only if the space of affine continuous functions on it with the supremum norm has the finite binary intersection property (F.2.I.P.) that is, if every finite collection of mutually intersecting closed balls has a nonvoid intersection (cf. [15]). By this and by [15, Theorem 4.7] any Banach space with the F.2.I.P. and at least one extreme point on its unit ball can be represented by the space of affine continuous functions on a suitable simplex. In $\S 3$ we prove a theorem concerning simultaneous extension for such spaces which is a generalization of the Borsuk-Dugundji-Arens extension theorem (cf. [4], [7], [3]).

The F.2.I.P. plays an important role in the theory of extension of compact operators. Lindenstrauss proved that a Banach space $X$ has the F.2.I.P. if and only if $X^{*}$ is an $L$-space or, if and only if any compact operator with the range in $X$ has an "almost" norm preserving extension (see [15, Theorem 6.1] for a precise formulation of this result). In [15] some criteria for the existence of norm preserving extensions of compact operators are proved as well as examples which show that in general a norm preserving extension may fail to exist. In $\$ 4$ we bring two other criteria when the range space has at least one extreme point on its closed unit ball (i.e., the range is the space of affine continuous functions on a simplex with the supremum norm). Examples given in $\$ 5$ show that in a certain sense these criteria are the best possible.

In the appendix of this paper we prove a theorem concerning the existence of continuous nearest point maps from $l_{1}$ onto its one-dimensional subspaces. This lemma is needed in the construction of one of the examples given in $\$ 5$.

All the linear spaces considered are over the reals. We follow the standard terminology and notations of [6] and [8].

If $X$ is a Banach space, $x \in X$ and $r>0$ then we denote by $S_{X}(x, r)$ the closed ball of $X$ with the center in $x$ and the radius $r$. The closed unit ball of $X$ is denoted also by $S_{X}$. When we refer to the topology of $X$ we mean its norm topology if no other topology is specified.

If $M$ is a set, $1_{M}$ denotes the function identically 1 on $M$. If $M \supset N$ and $f$ is a function defined on $M,\left.f\right|_{N}$ denotes the restriction of $f$ to $N$. By $2^{M}$ we denote the family of all nonvoid subsets of $M$.

In the sequel we assume, unless stated otherwise, that $K$ is a simplex. A subset $F$ of $K$ is called a face of $K$ if it is convex and if the relations $0<\lambda<1, k_{1}, k_{2} \in K$, $\lambda k_{1}+(1-\lambda) k_{2} \in F$ imply $k_{1}, k_{2} \in F$. By $\partial K$ we denote the set of extreme points of $K$. $A(K)$ (and if no confusion is possible just $A$ ) is the space of affine continuous functions on $K$ with the supremum norm.

If we define $T: K \rightarrow A^{*}$ by $T k(f)=f(k)$ for every $k \in K$ and $f \in A$ then $T$ is an affine homeomorphism and it is often convenient to consider $K$ embedded in this way in $A^{*}$. 
$K$ is called an $r$-simplex (cf. [1]) if $\partial K$ is closed. For an $r$-simplex $A(K)=C(\partial K)$ and conversely, any space of continuous functions on a compact Hausdorff space can be represented as the space of affine continuous functions on an $r$-simplex.

2. A collection of nonnegative functions $\left\{\psi_{j}\right\}_{j=1}^{m} \subset A$ is called a partition of unity on $K$ if $\sum_{j=1}^{m} \psi_{j}=1_{K}$. In [13] we proved the existence of certain paritions of unity on simplexes. The corollary of the subsequent lemma provides us with another type of partitions of unity which is more adequate to our purposes here.

LEMMA 2.1. Let $\left\{f_{i}\right\}_{i=1}^{n},\left\{g_{i}\right\}_{i=1}^{n}$ be two subsets of $A$ and let $\left\{k_{l_{1}}\right\}_{l_{1}=1}^{p} \subset \partial K\left(k_{l_{1}} \neq k_{l_{2}}\right.$ if $l_{1} \neq l_{2}$ ). Assume that there are functions $\left\{\phi_{j}\right\}_{j=1}^{m}$ defined on $\partial K$ such that

$$
\left.f_{i}\right|_{\partial K} \leqq \sum_{j=1}^{m} \alpha_{i j} \phi_{j} \leqq\left. g_{i}\right|_{\partial K}, \quad 1 \leqq i \leqq n,
$$

for some scalars $\alpha_{i j}$ and let

$$
\phi_{j}\left(k_{l}\right)=\beta_{j l}, \quad 1 \leqq j \leqq m, 1 \leqq l \leqq p .
$$

Then there exist functions $\left\{\psi_{j}\right\}_{j=1}^{m} \subset A$ such that

$$
f_{i} \leqq \sum_{j=1}^{m} \alpha_{i j} \psi_{j} \leqq g_{i}, \quad 1 \leqq i \leqq n,
$$

(everywhere on $K$ ) and

$$
\psi_{j}\left(k_{l}\right)=\beta_{j l}, \quad 1 \leqq j \leqq m, 1 \leqq l \leqq p .
$$

Proof. We shall proceed by induction on $m$. Suppose that $m=1$ and we are given

$$
\begin{gathered}
\left.f_{i}\right|_{\partial K} \leqq \alpha_{i 1} \phi_{1} \leqq\left. g_{i}\right|_{\partial K}, \quad 1 \leqq i \leqq n, \\
\phi_{1}\left(k_{l}\right)=\beta_{1 l}, \quad 1 \leqq l \leqq p .
\end{gathered}
$$

Without loss of generality we may suppose $\alpha_{i 1} \geqq 0,1 \leqq i \leqq n$. Consider

$$
\begin{aligned}
& \mathscr{F}_{1}^{\prime}=\left\{\frac{1}{\alpha_{i 1}} f_{i}: \alpha_{i 1} \neq 0\right\}, \\
& \mathscr{F}_{1}^{\prime \prime}=\left\{\frac{1}{\alpha_{i 1}} g_{i}: \alpha_{i 1} \neq 0\right\} .
\end{aligned}
$$

By (5), on $\partial K$ any function of $\mathscr{F}_{1}^{\prime}$ is not greater than any function of $\mathscr{F}_{1}^{\prime \prime}$ and according to a known principle of maximum [5, Lemma 17] this is true for all $K$.

If we define

$$
h_{1}^{\prime}(k)=\max \left\{f(k): f \in \mathscr{F}_{1}^{\prime}\right\}, \quad h_{1}^{\prime \prime}=\min \left\{f(k): f \in \mathscr{F}_{1}^{\prime \prime}\right\}, \quad k \in K,
$$

then $h_{1}^{\prime},-h_{1}^{\prime \prime}$ are convex continuous functions on $K$ and $h_{1}^{\prime} \leqq h_{1}^{\prime \prime}$. Moreover, $h_{1}^{\prime}\left(k_{l}\right)$ $\leqq \beta_{1 l} \leqq h_{1}^{\prime \prime}\left(k_{l}\right), 1 \leqq l \leqq p$. Since co $\left(k_{1}, \ldots, k_{l}\right)$ is a closed face of $K$, a theorem of 
Edwards [9, p. 12] yields a function $\psi_{1} \in A$ such that $\psi_{1}\left(k_{l}\right)=\beta_{1 l}, 1 \leqq l \leqq p$, and $h_{1}^{\prime} \leqq \psi_{1} \leqq h_{1}^{\prime \prime}$. Then clearly $\psi_{1}$ satisfies $f_{i} \leqq \alpha_{i 1} \psi_{1} \leqq g_{i}$ whenever $\alpha_{i 1} \neq 0$ and by (5),

$$
\left.f_{i}\right|_{\partial K} \leqq\left.\alpha_{i 1} \psi_{1}\right|_{\partial K} \leqq\left. g_{i}\right|_{\partial K}
$$

whenever $\alpha_{i 1}=0$. The above quoted principle of maximum implies that (6) holds everywhere on $K$ and this completes the first step of the proof.

Suppose now that the lemma is true for $m-1$ functions and we are given (1) and (2). As before we assume $\alpha_{i m} \geqq 0,1 \leqq i \leqq n$. From (1) we get

$$
\frac{1}{\alpha_{i m}} f_{i}-\sum_{j=1}^{m-1} \frac{\alpha_{i j}}{\alpha_{i m}} \phi_{j} \leqq \phi_{m} \leqq \frac{1}{\alpha_{i m}} g_{i}-\sum_{j=1}^{m-1} \frac{\alpha_{i j}}{\alpha_{i m}} \phi_{j}, \quad \text { on } \partial K,
$$

whenever $\alpha_{i m} \neq 0$. For any $r$ and $s$ such that $\alpha_{r m} \neq 0 \neq \alpha_{s m}$ we have

$$
\frac{1}{\alpha_{r m}} f_{r}-\frac{1}{\alpha_{s m}} g_{s} \leqq \sum_{j=1}^{m-1}\left(\frac{\alpha_{r j}}{\alpha_{r m}}-\frac{\alpha_{s j}}{\alpha_{s m}}\right) \phi_{j} \leqq \frac{1}{\alpha_{r m}} g_{r}-\frac{1}{\alpha_{s m}} f_{s}, \quad \text { on } \partial K \text {. }
$$

The functions $\phi_{1}, \ldots, \phi_{m-1}$ satisfy also

$$
\left.f_{i}\right|_{\partial K} \leqq \sum_{j=1}^{m-1} \alpha_{i j} \phi_{j} \leqq\left. g_{i}\right|_{\partial K}
$$

if $\alpha_{i m}=0$.

Applying the induction hypothesis to (8) or (9), we can find $\left\{\psi_{j}\right\}_{j=1}^{m-1} \subset A$ such that

$$
\begin{gathered}
\psi_{j}\left(k_{l}\right)=\beta_{j l}, \quad 1 \leqq j \leqq m-1,1 \leqq l \leqq p, \\
\frac{1}{\alpha_{r m}} f_{r}-\sum_{j=1}^{m-1} \frac{\alpha_{r j}}{\alpha_{r m}} \psi_{j} \leqq \frac{1}{\alpha_{s m}} g_{s}-\sum_{j=1}^{m-1} \frac{\alpha_{s j}}{\alpha_{s m}} \psi_{j}
\end{gathered}
$$

whenever $\alpha_{r m} \neq 0 \neq \alpha_{s m}$ and

$$
f_{i} \leqq \sum_{j=1}^{m-1} \alpha_{i j} \psi_{j} \leqq g_{i}
$$

if $\alpha_{i m}=0$. Using Edwards' theorem in the same way as above we obtain the function $\psi_{m}$ which satisfies (3) and (4).

COROLLARY 2.2. Let $\left\{f_{i}\right\}_{i=1}^{m} \subset A$ and $\varepsilon>0$. Suppose that $\left\{\phi_{j}\right\}_{j=1}^{m}$ are nonnegative functions defined on $\partial K,\left\{k_{j}\right\}_{j=1}^{m} \subset \partial K$ and $a_{i j}, 1 \leqq i \leqq n, 1 \leqq j \leqq m$, are real numbers such that:

(i) $\sum_{j=1}^{m} \phi_{j}=1$;

(ii) $\phi_{j}\left(k_{l}\right)=\delta_{j}^{l}, 1 \leqq j \leqq m, 1 \leqq l \leqq m$;

(iii) $\left|f_{i}(k)-\sum_{j=1}^{m} a_{i j} \phi_{j}(k)\right| \leqq \varepsilon$ for any $k \in \partial K, 1 \leqq i \leqq n$.

Then there exists a partition of unity $\left\{\psi_{j}\right\}_{j=1}^{m} \subset A$ such that:

(iv) $\psi_{j}\left(k_{l}\right)=\delta_{j}^{l}, 1 \leqq j \leqq m, 1 \leqq l \leqq m ;$

(v) $\left|f_{i}(k)-\sum_{j=1}^{m} a_{i j} \psi_{j}(k)\right| \leqq \varepsilon$ for every $k \in K, 1 \leqq i \leqq n$. 
Proof. The functions $\phi_{1}, \ldots, \phi_{m-1}$ satisfy the relations:

(1) $f_{i}-\varepsilon-a_{i m} \leqq \sum_{j=1}^{m-1}\left(a_{i j}-a_{i m}\right) \phi_{j} \leqq f_{i}+\varepsilon-a_{i m}, 1 \leqq i \leqq n$, on $\partial K$,

(2) $0 \leqq \sum_{j=1}^{m-1} \phi_{j} \leqq 1$,

(3) $0 \leqq \phi_{j} \leqq 1,1 \leqq j \leqq m-1$,

(4) $\phi_{j}\left(x_{l}\right)=\delta_{j}^{l}, 1 \leqq j \leqq m-1,1 \leqq l \leqq m$.

By the preceding lemma there exists $\psi_{1}, \ldots, \psi_{m-1}$ in $A$ such that (1)-(4) are valid when $\psi_{j}$ stand instead of $\phi_{j}$. Defining $\psi_{m}=1-\sum_{j=1}^{m-1} \psi_{j}$ we obtain a partition of unity on $K$ which obviously satisfies (iv) and (v).

LEMMA 2.3. Let $E$ be a linear topological space and $T$ an affine lower semicontinuous map from $K$ to $2^{E}$. Let $f$ be a continuous linear functional on $E$.

(a) If $f^{*}(k)=\sup _{x \in T(k)} f(x), f_{*}(k)=\inf _{x \in T(k)} f(x)$ then $f^{*},-f_{*}$ are concave lower semicontinuous (single-valued) functions on $K\left(f^{*}: K \rightarrow(-\infty, \infty], f_{*}: K \rightarrow[-\infty, \infty)\right.$ ).

(b) Let $f^{\prime} \in A$ with $f_{*} \leqq f^{\prime} \leqq f^{*}$ and $\varepsilon>0$. The map $T^{\prime}: K \rightarrow 2^{E}$ defined by

$$
T^{\prime}(k)=T(k) \cap f^{-1}\left[\left(f^{\prime}(k)-\varepsilon, f^{\prime}(k)+\varepsilon\right)\right]
$$

is affine and lower semicontinuous.

Proof. (a) It is enough to prove the assertion for $f^{*}$. Pick $k_{1}, k_{2} \in K, \lambda \in[0,1]$. Then

$$
\begin{aligned}
f^{*}\left(\lambda k_{1}+(1-\lambda) k_{2}\right) & =\sup \left\{f(x): x \in T\left(\lambda k_{1}+(1-\lambda) k_{2}\right)\right\} \\
& \geqq \sup \left\{f(x): x \in \lambda T\left(k_{1}\right)+(1-\lambda) T\left(k_{2}\right)\right\} \\
& =\lambda \sup _{x \in T\left(k_{1}\right)} f(x)+(1-\lambda) \sup _{x \in T\left(k_{2}\right)} f(x) \\
& =\lambda f^{*}\left(k_{1}\right)+(1-\lambda) f^{*}\left(k_{2}\right)
\end{aligned}
$$

which means that $f^{*}$ is concave.

Let $a$ be any real number. Obviously,

$$
\begin{aligned}
\left\{k \in K ; f^{*}(k)>a\right\} & =\{k \in K: \exists x \in T(k), f(x)>a\} \\
& =\left\{k \in K: T(k) \cap f^{-1}((a, \infty)) \neq \varnothing\right\} .
\end{aligned}
$$

$T$ being lower semicontinuous the last set is open in $K$ and this implies that $f^{*}$ is lower semicontinuous.

(b) Clearly $T^{\prime}(k)$ is a convex nonvoid subset of $E$ for every $k \in K$. Let us show that

(1) $\lambda T^{\prime}\left(k_{1}\right)+(1-\lambda) T^{\prime}\left(k_{2}\right) \subset T^{\prime}\left(\lambda k_{1}+(1-\lambda) k_{2}\right)$ for any $k_{1}, k_{2} \in K, 0<\lambda<1$.

Pick $x_{i} \in T^{\prime}\left(k_{i}\right), i=1,2$. Then $x_{i} \in T\left(k_{i}\right)$ and $f^{\prime}\left(k_{i}\right)-\varepsilon<f\left(x_{i}\right)<f^{\prime}\left(k_{i}\right)+\varepsilon$.

Since $f, f^{\prime}$ and $T$ are affine we have

$$
f^{\prime}\left(\lambda k_{1}+(1-\lambda) k_{2}\right)-\varepsilon<f\left(\lambda x_{1}+(1-\lambda) x_{2}\right)<f^{\prime}\left(\lambda k_{1}+(1-\lambda) k_{2}\right)+\varepsilon
$$

and

Hence

$$
\lambda x_{1}+(1-\lambda) x_{2} \in T\left(\lambda k_{1}+(1-\lambda) k_{2}\right) .
$$

and this proves (1).

$$
\lambda x_{1}+(1-\lambda) x_{2} \in T^{\prime}\left(\lambda k_{1}+(1-\lambda) k_{2}\right)
$$


To prove that $T^{\prime}$ is lower semicontinuous we use a device of Michael [18]. Let $U$ be an open subset of $E$. We have to show that $V=\left\{k \in K: T^{\prime}(k) \cap U \neq \varnothing\right\}$ is open in $K$. Let $k_{0} \in V$ and $x_{0} \in T^{\prime}\left(k_{0}\right) \cap U$. Then $x_{0} \in T\left(k_{0}\right) \cap U$ and $f^{\prime}\left(k_{0}\right)-\varepsilon<f\left(x_{0}\right)$ $<f^{\prime}\left(k_{0}\right)+\varepsilon$.

Choose $\varepsilon^{\prime}$ such that $0<\varepsilon^{\prime}<\varepsilon$ and $f^{\prime}\left(k_{0}\right)-\varepsilon^{\prime}<f\left(x_{0}\right)<f^{\prime}\left(k_{0}\right)+\varepsilon^{\prime}$. The sets

$$
\begin{aligned}
& W_{1}=\left\{k \in K: T(k) \cap U \cap f^{-1}\left[\left(f^{\prime}\left(k_{0}\right)-\varepsilon^{\prime}, f^{\prime}\left(k_{0}\right)+\varepsilon^{\prime}\right)\right] \neq \varnothing\right\}, \\
& W_{2}=\left\{k \in K: f^{\prime}\left(k_{0}\right)-\varepsilon+\varepsilon^{\prime}<f^{\prime}(k)<f^{\prime}\left(k_{0}\right)+\varepsilon-\varepsilon^{\prime}\right\}
\end{aligned}
$$

are open in $K$ and $k_{0} \in W_{1} \cap W_{2}$. The proof will be achieved if we show that $W_{1} \cap W_{2} \subset V$.

Pick $k \in W_{1} \cap W_{2}$. By the definition of $W_{1}, W_{2}$ there exists $x \in T(k) \cap U$ such that

$$
f^{\prime}(k)-\varepsilon<f^{\prime}\left(k_{0}\right)-\varepsilon^{\prime}<f(x)<f^{\prime}\left(k_{0}\right)+\varepsilon^{\prime}<f^{\prime}(k)+\varepsilon .
$$

Hence $x \in T^{\prime}(k) \cap U$. We showed that for any $k \in W_{1} \cap W_{2}$ the set $T^{\prime}(k) \cap U \neq \varnothing$ i.e., $W_{1} \cap W_{2} \subset V$. This concludes the proof of the lemma.

If $E$ is a metrizable locally convex linear topological space then it is well known that there is a nondecreasing sequence of seminorms on $E, p_{1} \leqq p_{2} \leqq \cdots \leqq p_{n} \leqq \cdots$, which defines the topology of the space. A metric on $E$ compatible with its topology and invariant with respect to translations is:

$$
\rho(x, y)=\sum_{n=1}^{\infty} \frac{1}{2^{n}} \cdot \frac{p_{n}(x-y)}{1+p_{n}(x-y)}, \quad x, y \in E .
$$

In the next lemma we shall use this metric as well as the following notation: if $x \in E$ and $r>0$ then

$$
\begin{aligned}
R(r, x) & =\{y \in E: \rho(x, y)<r\}, \\
R^{n}(r, x) & =\left\{y \in E: p_{n}(x-y)<r\right\} .
\end{aligned}
$$

LEMMA 2.4. Let $E$ be a metrizable locally convex linear topological space, $T: K \rightarrow 2^{E}$ an affine lower semicontinuous map and $\varepsilon>0$. There exist an affine lower semicontinuous transformation $T^{\prime}: K \rightarrow 2^{E}$, a partition of unity on $K,\left\{\psi_{j}\right\}_{j=1}^{m} \subset A, a$ subset $\left\{k_{j}\right\}_{j=1}^{m} \subset \partial K$ and a subset $\left\{y_{j}\right\}_{j=1}^{m} \subset E$ such that $\psi_{j}\left(k_{j}\right)=1,1 \leqq j \leqq m$, and

(1) $T^{\prime}(k) \subset T(k) \cap R\left(\varepsilon, \sum_{j=1}^{m} \psi_{j}(k) y_{j}\right)$ for any $k \in K$.

Proof. Choose an integer $N$ such that

(2) $\sum_{n=N+1}^{\infty} 1 / 2^{n}<\varepsilon / 8$.

For any $x \in E$ put $U_{x}=\left\{k \in K: T(k) \cap R^{N}(\varepsilon / 8, x) \neq \varnothing\right\}$. The family $\left\{U_{x}\right\}_{x \in E}$ is an open covering of $K$. Let $U_{x_{1}}, \ldots, U_{x_{p}}$ be a finite subcovering extracted from it. Denote

$$
C=R^{N}(\varepsilon / 8,0)+\operatorname{co}\left(x_{1}, \ldots, x_{p}\right) \text {. }
$$

$C$ is an open convex subset of $E$ and for any $k \in K, C \cap T(k) \neq \varnothing$. It is easy to see 
that the map $T_{0}: K \rightarrow 2^{E}$ defined by $T_{0}(k)=C \cap T(k)$ is affine and lower semicontinuous.

Let

$$
D=C-\operatorname{co}\left(x_{1}, \ldots, x_{p}\right)=R^{N}(\varepsilon / 8,0)+\operatorname{co}\left(x_{1}, \ldots, x_{p}\right)-\operatorname{co}\left(x_{1}, \ldots, x_{p}\right) .
$$

Since co $\left(x_{1}, \ldots, x_{p}\right)-\operatorname{co}\left(x_{1}, \ldots, x_{p}\right)$ is a compact subset of $E$, by a well-known consequence of the Hahn-Banach theorem we can find $\left\{f_{i}\right\}_{i=1}^{n} \subset E^{*}$ such that

(3) $p_{N}(x)-\varepsilon / 2 \leqq \max _{1 \leqq i \leqq n}\left|f_{i}(x)\right| \leqq p_{N}(x)$

for any $x \in D$.

Define, for $k \in K$,

$$
\left(f_{1}\right)^{*}(k)=\sup _{x \in T_{0}(k)} f_{1}(x), \quad\left(f_{1}\right)_{*}(k)=\inf _{x \in T_{0}(k)} f_{1}(x) .
$$

By Lemma $2.3\left(f_{1}\right)^{*}$ and $\left(-f_{1}\right)_{*}$ are concave lower semicontinuous functions. From a theorem of Edwards [9, Theorem 3] it follows that there exists $f_{1}^{\prime} \in A$ such that $\left(f_{1}\right)_{*} \leqq f_{1}^{\prime} \leqq\left(f_{1}\right)^{*}$. By Lemma 2.3 the transformation

(4) $T_{1}(k)=T_{0}(k) \cap f_{1}^{-1}\left[\left(f_{1}^{\prime}(k)-\varepsilon / 8, f_{1}^{\prime}(k)+\varepsilon / 8\right)\right]$

is affine and lower semicontinuous. Clearly, if $x \in T_{1}(k)$ then $\left|f_{1}(x)-f_{1}^{\prime}(k)\right|<\varepsilon / 8$.

Let $i$ be a natural number, $1 \leqq i<n$. Suppose that we have found $T_{i}: K \rightarrow 2^{E}$ affine and lower semicontinuous and $\left\{f_{l}^{\prime}\right\}_{l=1}^{i} \subset A$ such that for any $k \in K, T_{i}(k)$ $\subset T_{0}(k)$ and if $x \in T_{i}(k)$ then $\left|f_{l}(x)-f_{l}^{\prime}(k)\right|<\varepsilon / 8,1 \leqq l \leqq i$. Using the functions

$$
\left(f_{i+1}\right)^{*}(k)=\sup _{x \in T_{i}(k)} f_{i+1}(x),\left(f_{i+1}\right)_{*}(k)=\inf _{x \in T_{i}(k)} f_{i+1}(x)
$$

in the same manner as we did for $\left(f_{1}\right)^{*}$ and $\left(f_{1}\right)_{*}$ we get $f_{i+1}^{\prime} \in A$ such that the map

$$
T_{i+1}(k)=T_{i}(k) \cap f_{i+1}^{-1}\left[\left(f_{i+1}^{\prime}(k)-\varepsilon / 8, f_{i+1}^{\prime}(k)+\varepsilon / 8\right)\right]
$$

is affine and lower semicontinuous. Clearly, if $x \in T_{i+1}(k)$ then $\left|f_{l}(x)-f_{l}^{\prime}(k)\right|<\varepsilon / 8$, $1 \leqq l \leqq i+1$.

Finally, we get an affine and lower semicontinuous transformation $\left(T_{n}=\right) T^{\prime}$ : $K \rightarrow 2^{E}$ and functions $\left\{f_{i}^{\prime}\right\}_{i=1}^{n} \subset A$ such that $T^{\prime}(k) \subset T_{0}(k) \subset T(k)$ for any $k \in K$ and if $x \in T^{\prime}(k)$ then

(5) $\left|f_{i}(x)-f_{i}^{\prime}(k)\right|<\varepsilon / 8,1 \leqq i \leqq n$.

Define for any $y \in \operatorname{co}\left(x_{1}, \ldots, x_{p}\right)$ the set

$$
V_{y}=\left\{k \in \operatorname{cl}(\partial K): T^{\prime}(k) \cap R^{N}(\varepsilon / 8, y) \neq \varnothing\right\} .
$$

$V_{y}$ is relatively open in $\mathrm{cl}(\partial K)$ and from $T^{\prime}(k) \subset T_{0}(k) \subset C$ it follows that the family $\left\{V_{y}\right\}_{y \in \operatorname{co}\left(x_{1}, \ldots, x_{p}\right)}$ is a covering of $\mathrm{cl}(\partial K)$. Choose a finite subcovering: $V_{y_{1}}, \ldots, V_{y_{m}}\left(\left\{y_{j}\right\}_{j=1}^{m} \subset \operatorname{co}\left(x_{1}, \ldots, x_{p}\right)\right)$. The sets $V_{y_{j}}^{\prime}=V_{y_{j}} \cap \partial K, 1 \leqq j \leqq m$, cover $\partial K$ and we can assume without loss of generality that for any $j, 1 \leqq j \leqq m$, there is $k_{j} \in V_{y_{j}}^{\prime}$ such that $k_{j} \notin V_{y_{l}}^{\prime}$ if $l \neq j$.

Define on $\partial K$ the following functions:

$$
\begin{aligned}
\phi_{j}(k)=1 & \text { if } j=\min \left\{l: k \in V_{y_{l}}^{\prime}\right\}, \\
& =0 \quad \text { if } j \neq \min \left\{l: k \in V_{y_{l}}^{\prime}\right\}, \quad 1 \leqq j \leqq m .
\end{aligned}
$$


Clearly $\phi_{j} \geqq 0, \phi_{j}\left(k_{l}\right)=\delta_{j}^{l}$ and $\sum_{j=1}^{m} \phi_{j}=1$. Moreover, for any $k \in \partial K$ there is one index $j_{k}$ and only one such that $\phi_{j_{k}}(k) \neq 0$. For this index $k \in V_{j_{k}}^{\prime}$. Hence there exists $x_{k} \in T^{\prime}(k)$ such that $p_{N}\left(x_{k}-y_{j_{k}}\right)<\varepsilon / 8$. This inequality can also be written in the following form:

$$
p_{N}\left(x_{k}-\sum_{j=1}^{m} \phi_{j}(k) y_{j}\right)<\frac{\varepsilon}{8}
$$

By (3)

$$
\left|f_{i}\left(x_{k}\right)-\sum_{j=1}^{m} f_{i}\left(y_{j}\right) \phi_{j}(k)\right|<\frac{\varepsilon}{8}, \quad 1 \leqq i \leqq n
$$

(since $x_{k}-\sum_{j=1}^{m} \phi_{j}(k) y_{j} \in D$ ).

From this and (5) it follows that for any $k \in K$ and $1 \leqq i \leqq n$ we have

$$
\left|f_{i}^{\prime}(k)-\sum_{j=1}^{m} f_{i}\left(y_{j}\right) \phi_{j}(k)\right| \leqq\left|f_{i}^{\prime}(k)-f_{i}\left(x_{k}\right)\right|+\left|f_{i}\left(x_{k}\right)-\sum_{j=1}^{m} f_{i}\left(y_{j}\right) \phi_{j}(k)\right| \leqq \frac{\varepsilon}{4}
$$

The Corollary 2.2 yields us a partition of unity $\left\{\psi_{j}\right\}_{j=1}^{m} \subset A$ such that $\psi_{j}\left(k_{l}\right)=\delta_{j}^{l}$, $1 \leqq j, l \leqq m$, and

$$
\left|f_{i}^{\prime}(k)-\sum_{j=1}^{m} f_{i}\left(y_{j}\right) \psi_{j}(k)\right| \leqq \frac{\varepsilon}{8}, \quad 1 \leqq i \leqq n, k \in K .
$$

We are going to show that $T^{\prime}$ and $\left\{\psi_{j}\right\}_{j=1}^{m}$ fulfill the conclusion of the lemma. We have only to prove that for any $k \in K$

(6) $T^{\prime}(k) \subset R\left(\varepsilon, \sum_{j=1}^{m} \psi_{j}(k) y_{j}\right)$.

Pick $x \in T^{\prime}(k)$. Then, by (5)

$$
\begin{aligned}
\left|f_{i}(x)-\sum_{j=1}^{m} f_{i}\left(y_{j}\right) \psi_{j}(k)\right| \leqq & \left|f_{i}(x)-f_{i}^{\prime}(k)\right| \\
& +\left|f_{i}^{\prime}(k)-\sum_{j=1}^{m} f_{i}\left(y_{j}\right) \psi_{j}(k)\right| \leqq \frac{\varepsilon}{4}+\frac{\varepsilon}{8}=\frac{3 \varepsilon}{8}, \quad 1 \leqq i \leqq n .
\end{aligned}
$$

Using (3) again we get

$$
p_{N}\left(x-\sum_{j=1}^{m} \psi_{j}(k) y_{j}\right) \leqq \frac{3 \varepsilon}{8}+\frac{\varepsilon}{2}=\frac{7 \varepsilon}{8} .
$$

This together with (2) and $p_{1} \leqq p_{2} \leqq \cdots \leqq p_{N}$ yields

$$
\rho\left(x, \sum_{j=1}^{m} \psi_{j}(k) y_{j}\right)<\frac{7 \varepsilon}{8} \sum_{n=1}^{N} \frac{1}{2^{n}}+\frac{\varepsilon}{8}<\varepsilon .
$$

Hence (6) is proved and this concludes the proof of the lemma.

3. Now we are going to prove a theorem which is the adaptation for simplexes of a well-known selection theorem of Michael [17], [18]. 
THEOREM 3.1. Let $E$ be a Fréchet space and $T: K \rightarrow 2^{E}$ an affine lower semicontinuous map such that $T(k)$ is closed for every $k \in K$. Then there exists an affine continuous selection for $T$ i.e. an affine continuous function $f: K \rightarrow E$ with $f(k) \in T(k)$ for each $k \in K$.

Proof. We assume that $E$ is equipped with the metric used in Lemma 2.4 and that $R(r, x)$ stands for $\{y \in E: \rho(x, y)<r\}$ if $x \in E$ and $r>0$. We define inductively a sequence of affine lower semicontinuous maps $T_{n}: K \rightarrow 2^{E}$ and a sequence of affine continuous functions $f_{n}: K \rightarrow E$ such that for every $k \in K, T_{n+1}(k) \subset T_{n}(k)$ $\subset T(k)$ and $T_{n}(k) \subset R\left(1 / 2^{n}, f_{n}(k)\right), n=1,2, \ldots$

$T_{1}$ and $f_{1}$ are those given by Lemma 2.4. Suppose that suitable $T_{1}, \ldots, T_{n}$ and $f_{1}, \ldots, f_{n}$ have already been chosen. From Lemma 2.4 we deduce the existence of an affine lower semicontinuous map $T_{n+1}: K \rightarrow 2^{E}$ and of an affine continuous function $f_{n+1}: K \rightarrow E$ such that for any $k \in K$,

$$
T_{n+1}(k) \subset T_{n}(k) \cap R\left(1 / 2^{n+1}, f_{n+1}(k)\right) .
$$

Thus the existence of the desired sequences is proved.

Now, $\left\{T_{n}(k)\right\}_{n=1}^{\infty}$ is a nonincreasing sequence of nonvoid sets with diameters tending to 0 . Hence $\bigcap_{n=1}^{\infty} \mathrm{cl}\left(T_{n}(k)\right)$ contains a point of $E$ and only one. Define for every $k \in K$

$$
f(k)=\bigcap_{n=1}^{\infty} \mathrm{cl}\left(T_{n}(k)\right) .
$$

Clearly $f(k) \in T(k)$ since $T(k)$ is closed and $T_{n}(k) \subset T(k)$. We have also that

$$
\rho\left(f(k), f_{n}(k)\right) \leqq 1 / 2^{n}
$$

for any $k \in K$ which means that the sequence $\left\{f_{n}\right\}_{n=1}^{\infty}$ converges uniformly to $f$. Hence $f$ is affine and continuous and this concludes the proof of the theorem.

One naturally asks the question if it is possible to use Michael's selection theorem mentioned above in order to obtain a shorter proof of Theorem 3.1. For instance, if $\partial K$ is Borel measurable one may pick a continuous selection $g$ for $\left.T\right|_{\partial_{K}}$ and extend it by defining $f(k)$ to be the integral of $g$ with respect to the representing measure of $k$. Indeed, if $\partial K$ is closed then this procedure leads to an affine continuous selection for $T$ (see [2]). On the other hand, as shown by the following example, in the general case this extension of $g$ may be discontinuous.

EXAMPLE 3.2. Let $A$ be the space of convergent sequences of real numbers $x=\{x(n)\}_{n=1}^{\infty}$ which satisfy $\lim _{n \rightarrow \infty} x(n)=\frac{1}{2}(x(1)+x(2))$ with the usual supremum norm. It is known that there is a simplex $K$ such that $A=A(K)$ (cf. $[15$, p. 78$]$ and the remark made in $\$ 1$ about the representation of spaces with the F.2.I.P. having an extreme point on the closed unit ball). For instance, $K$ may be the positive part of the unit sphere of $A^{*}\left(=l_{1}\right)$ when $A$ is ordered by $x \geqq 0 \Leftrightarrow x(n) \geqq 0, n=1,2, \ldots$ and $A^{*}$ is equipped with the dual order. The extreme points of $K$ are the functionals $k_{n}(x)=x(n)$ and in its topology (the $w^{*}$-topology of $\left.A^{*}\right) k_{n} \rightarrow \frac{1}{2}\left(k_{1}+k_{2}\right)$. Consider 
the map: $T(k)=[0,1], k \in K$. Clearly $T$ is an affine lower semicontinuous map from $K$ to $2^{(-\infty, \infty)}$. Choose the following continuous selection for $\left.T\right|_{\partial_{K}}: g\left(k_{n}\right)=1 / n$. The extension of $g$ described above is

$$
F(k)=\sum_{n=1}^{\infty} \frac{a_{n}}{n}, \quad k=\left(a_{1}, a_{2}, \ldots\right) \in K .
$$

Obviously $f$ is discontinuous at $\frac{1}{2}\left(k_{1}+k_{2}\right)$.

COROllaRY 3.3. Let $K$ be a compact convex subset of a locally convex linear topological space. The following statements are equivalent:

(i) $K$ is a simplex.

(ii) If $E$ is a Fréchet space and $T: K \rightarrow 2^{E}$ is an affine lower semicontinuous map with $T(k)$ closed for every $k \in K$ then $T$ admits an affine continuous selection.

(iii) If $T: K \rightarrow 2^{(-\infty, \infty)}$ is an affine lower semicontinuous map and $T(k)$ is closed for every $k \in K$ then $T$ admits an affine continuous selection.

Proof. (i) $\Rightarrow$ (ii) has already been proved and (ii) $\Rightarrow$ (iii) is trivial. Suppose (iii) is true. According to [5, Théorème 11] it is enough to show that for any convex and continuous function $f$ given on $K$ the function $\hat{f}: K \rightarrow(-\infty, \infty)$ defined by

$$
\hat{f}(k)=\inf \{g(k): g \geqq f, g \in A(K)\}\left(^{2}\right)
$$

is affine. This will become clear if we observe that the set $\{g: g \geqq f, g \in A(K)\}$ is directed by $\geqq$. Indeed, let $g_{1}, g_{2} \geqq f, g_{i} \in A(K)$ and put $h=\min \left(g_{1}, g_{2}\right)$. Then $h$ is continuous and concave. Hence $T(k)=[f(k), h(k)]$ is an affine lower semicontinuous map from $K$ to $2^{(-\infty, \infty)}$. If $g \in A(K)$ is a selection for $T$ then $f \leqq g \leqq h$ and this completes the proof.

Corollary 3.4. Let $E$ and $T$ be as in Theorem 3.1. If $F$ is a closed face of the simplex $K$ and $f: F \rightarrow E$ is an affine continuous selection for $\left.T\right|_{F}$ then there exists an affine continuous selection for $T$, say $\phi$, such that $\left.\phi\right|_{F}=f$.

Proof. The map $T^{\prime}: K \rightarrow 2^{E}$ given by

$$
\begin{aligned}
T^{\prime}(k) & =\{f(k)\} & & \text { if } k \in F, \\
& =T(k) & & \text { if } k \in K \sim F,
\end{aligned}
$$

satisfies the conditions of Theorem 3.1 and $T^{\prime}(k) \subset T(k)$ for each $k \in K$. Any affine continuous selection of $T^{\prime}$ is suitable.

The subsequent theorem reduces for metrizable $r$-simplexes to a particular case of Dugundji's extension theorem [7].

THEOREM 3.5. Let $E$ be a Fréchet space and $F$ a closed face of the simplex $K$. If $f: F \rightarrow E$ is affine and continuous there exists $\Phi: K \rightarrow E$ with $\left.\Phi\right|_{F}=f$ and $\Phi(K)=f(F)$.

$\left.{ }^{2}\right)$ Here we denote by $A(K)$ the space of all affine continuous functions on the compact convex set $K$. 
Proof. Define $T: K \rightarrow 2^{E}$ by $T(k)=f(F)$ for every $k \in K$ and apply Corollary 3.4. To see how this is related to Dugundji's theorem one can use two results of Alfsen: (1) if $K$ is an $r$-simplex and $M=\bar{M} \subset \partial K$ then co $\bar{M}$ is a closed face of $K$ [1]; (2) if $\Phi$ is a continuous function from $\partial K$ ( $K$ is an $r$-simplex) into a compact convex subset of a locally convex linear topological space then $\Phi$ has an affine continuous extension to $K[2]\left({ }^{3}\right)$.

THEOREM 3.6. Let $F$ be a metrizable closed face of the simplex $K$. Then there exists an affine and continuous map $\Phi: K \rightarrow F$ with $\Phi(k)=k$ for every $k \in F$.

Proof. The face $F$ is a simplex and the Banach space $A(F)$ is separable. Let $B=\left\{f_{n}\right\}_{n=1}^{\infty}$ be a dense subset of it. $B$ induces in $A^{*}(F)$ a metrizable locally convex topology $\tau$. This topology coincides with the $w^{*}$-topology on the canonical copy of $F$ in $A^{*}(F)$ (which will be identified with $F$ in the remainder of the proof). Hence $F$ is compact in its $\tau$-topology and remains compact (hence closed) in the completion of $\left(A^{*}(F), \tau\right)$. But the completion of $\left(A^{*}(F), \tau\right)$ is a Fréchet space and if we define

$$
\begin{aligned}
T(k) & =\{k\} & & \text { if } k \in F, \\
& =F & & \text { if } k \in K \sim F,
\end{aligned}
$$

we have an affine and lower semicontinuous map from $K$ into the family of closed convex sets of a Fréchet space. The selection of $T$ given by Theorem 3.1 satisfies the requirements.

The corollary stated below generalizes for simplexes the Borsuk-DugundjiArens extension theorem (cf. [4], [7], [3]).

COROLLARY 3.7. If $F$ is a metrizable closed face of the simplex $K$ then there exists a linear map $T$ from $A(F)$ into $A(K)$ with the following properties:

(i) if $k \in F$ then $T f(k)=f(k)$;

(ii) if $f \geqq 0$ then $T f \geqq 0$;

(iii) $T 1_{F}=1_{K}$;

(iv) $\|T f\|=\|f\|$.

There exists a projection of norm 1 of $A(K)$ onto the range of $T$ along the subspace of $A(K)$ consisting of all the functions which vanish everywhere on $F$.

Proof. If $\Phi: K \rightarrow F$ is the map given by the preceding theorem then the map $T$ defined by

$$
T f(k)=f(\Phi(k)), \quad f \in A(F), k \in K,
$$

has all the properties (i)-(iv) and is linear. If $U: A(K) \rightarrow A(F)$ is the restriction map

$$
U \phi=\left.\phi\right|_{F}, \quad \phi \in A(K),
$$

then $T \circ U$ is the desired projection.

$\left({ }^{3}\right)$ This is a corollary of the main theorem of [2]. Here is the place to remark that this theorem is valid for simplexes without the hypothesis of metrizability. 
4. The selection theorem of Michael mentioned above [17, Theorem 3.2] and its consequences have been very useful in problems of extension of compact operators (e.g. [15], [16]). We shall give here some applications of Theorem 3.1 in this direction. First we need a representation for operators having the range in $A$ and this is done by the next lemma whose proof is identical with that of the representation theorem for operators with the range in a space of continuous functions [8, p. 490].

LEMMA 4.1. Let $X$ be a Banach space and $T$ a linear bounded operator from $X$ to $A$. Then there exists an affine and $w^{*}$-continuous function $\chi: K \rightarrow X^{*}$ for which

(1) $T x(k)=\chi(k)(x), x \in X, k \in K$,

(2) $\|T\|=\sup _{k \in K}\|\chi(k)\|$.

Conversely, to any affine and $w^{*}$-continuous function from $K$ into $X^{*}$ there corresponds a linear bounded operator $T: X \rightarrow A$ given by (1) of which norm satisfies (2). $T$ is compact ( $w$-compact) if and only if $\chi$ is continuous in the norm topology ( $w$ topology) of $X^{*}$.

Our first result is a particular case of a known extension theorem [15, Theorem 6.1]. The proof given here is short and the pattern we chose will be useful later.

COROllaRY 4.2. Let $X \subset Y$ be Banach spaces and $T$ a compact operator from $X$ to $A$. For any $\varepsilon>0$ there exists a compact extension $\tilde{T}$ of $T$ from $Y$ to $A$ with $\|\tilde{T}\| \leqq(1+\varepsilon)\|T\|$.

Proof. Let $\phi$ be the restriction map from $Y^{*}$ to $X^{*}: \phi\left(y^{*}\right)=y^{*} \mid X$. Define $\Phi: S_{X^{*}}(0,\|T\|) \rightarrow 2^{Y^{*}}$ by

$$
\Phi\left(x^{*}\right)=\left\{y^{*} \in Y^{*}: \phi\left(y^{*}\right)=x^{*},\left\|y^{*}\right\| \leqq(1+\varepsilon)\|T\|\right\} .
$$

Obviously $\Phi$ is affine and $\Phi\left(x^{*}\right)$ is norm-closed for every $x^{*} \in S_{X^{*}}(0,\|T\|)$. We are going to show that $\Phi$ is lower semicontinuous. Let $U$ be an open subset of $Y^{*}$ and pick $x_{0}^{*} \in\left\{x^{*} \in S_{X^{*}}(0,\|T\|): \Phi\left(x^{*}\right) \cap U \neq \varnothing\right\}$ (of course, we assume that this set is not void; if it is void, nothing is to be proved). Since $\left\|x_{0}^{*}\right\| \leqq\|T\|$ and $U$ is open, there exists $y_{0}^{*} \in \Phi\left(x_{0}^{*}\right) \cap U$ with $\left\|y_{0}^{*}\right\|<(1+\varepsilon)\|T\|$. Let $V$ be an open neighborhood of $y_{0}^{*}$ contained in $U \cap\left\{y^{*} \in Y^{*}:\left\|y^{*}\right\|<(1+\varepsilon)\|T\|\right\}$. Since $\phi$ is an open map, $\phi(V)$ is open in $X^{*}$. Clearly

$$
x_{0}^{*} \in \phi(V) \cap S_{X^{*}}(0,\|T\|) \subset\left\{x^{*} \in S_{X^{*}}(0,\|T\|): \Phi\left(x^{*}\right) \cap U \neq \varnothing\right\},
$$

hence $\left\{x^{*} \in S_{X^{*}}(0,\|T\|): \Phi\left(x^{*}\right) \cap U \neq \varnothing\right\}$ is relatively open in $S_{X^{*}}(0,\|T\|)$ and our assertion about $\Phi$ is proved.

Let $\chi: K \rightarrow S_{X} \cdot(0,\|T\|)$ be the function given by Lemma 4.1 for $T$. From what we have proved above we infer that $\Phi \circ \chi$ fulfils the conditions of Theorem 3.1. Let $\tilde{\chi}: K \rightarrow S_{Y \circ}(0,(1+\varepsilon)\|T\|)$ be an affine continuous selection of $\Phi \circ \chi$. It is easily seen that the corresponding operator $\tilde{T}: Y \rightarrow A$ has the required properties.

In studying norm preserving extensions of compact operators the following concept has been found useful [15, p. 86]: if $X \subset Y$ are Banach spaces, a map $\psi$ 
from $X^{*}$ to $Y^{*}$ is called a continuous norm preserving extension (C.N.P.E.) map if it is continuous in the norm topologies of $X^{*}$ and $Y^{*}$ and satisfies

$$
\left.\psi\left(x^{*}\right)\right|_{x}=x^{*}, \quad\left\|\psi\left(x^{*}\right)\right\|=\left\|x^{*}\right\|, \quad x^{*} \in X^{*} .
$$

Lindenstrauss proved [15, Lemma 7.2(a)] that if there is a C.N.P.E. map from $X^{*}$ to $Y^{*}$ then every compact operator from $X$ to a $C(S)$ space has a compaci norm preserving extension from $Y$ to $C(S)$. An example given in $\$ 5$ shows that this assertion cannot be generalized for spaces of affine functions without some additional hypothesis.

If $X \subset Y$ are Banach spaces and each functional on $X$ has a unique norm preserving extension to $Y, X$ is called a $U$ subspace of $Y$ (cf. [19]).

THEOREM 4.3. Let $X$ be a $U$ subspace of $Y$ and suppose that the norm preserving extension of functionals on $X$ to $Y$ is norm continuous. Then every compact operator from $X$ to $A$ has a compact norm preserving extension from $Y$ to $A$.

Proof. The proof of the preceding result makes clear that it is enough to show that the map $\Phi: S_{X^{*}}(0,\|T\|) \rightarrow 2^{Y^{*}}$ defined by

$$
\Phi\left(x^{*}\right)=\left\{y^{*} \in Y^{*}:\left.y^{*}\right|_{x}=x^{*},\left\|y^{*}\right\| \leqq\|T\|\right\}
$$

is lower semicontinuous (it is obviously affine).

Denote by $\psi$ the norm preserving extension map from $X^{*}$ to $Y^{*}$ i.e., $\left.\psi\left(x^{*}\right)\right|_{X}=x^{*}$, $\left\|\psi\left(x^{*}\right)\right\|=\left\|x^{*}\right\|$. By our assumption $\psi$ is continuous. Let $V$ be an open subset of $Y^{*}$ and pick $x_{0}^{*} \in\left\{x^{*} \in S_{X^{*}}(0,\|T\|): \Phi\left(x^{*}\right) \cap V \neq \varnothing\right\}=M$. If $\left\|x_{0}^{*}\right\|<\|T\|$ then the same argument as in the proof of Corollary 4.2 will convince us that $x_{0}^{*}$ is an interior point of $M$. Suppose now that $\left\|x_{0}^{*}\right\|=\|T\|$. Then $\Phi\left(x_{0}^{*}\right)=\left\{\psi\left(x_{0}^{*}\right)\right\}$ and

$$
x_{0}^{*} \in \psi^{-1}(V) \cap S_{X^{*}}(0,\|T\|) \subset M .
$$

$\psi^{-1}(V)$ is open in $X^{*}$; therefore $x_{0}^{*}$ is an interior point of $M$ in the relative topology of $S_{X^{*}}(0,\|T\|)$ and this concludes the proof.

REMARK. By [19] and [15, p. 87-88] a typical case in which this theorem applies is when $Y^{*}$ is locally uniformly convex.

Now we are going to prove that the hypothesis that $X$ is a $U$ subspace of $Y$ can be discarded if $\operatorname{dim} T(X)=2$. However, as Example 5.3 shows, if $\operatorname{dim} T(X)=3$ a norm preserving extension may not exist if $X$ is not a $U$ subspace of $Y$.

LEMMA 4.4. Let $X \subset Y$ be Banach spaces and suppose that there is a C.N.P.E. map $\psi$ from $X^{*}$ to $Y^{*}$. Let $Z$ be a two-dimensional subspace of $X^{*}$. There exists an affine lower semicontinuous map $\Phi: S_{Z} \rightarrow 2^{Y^{*}}$ such that for every $x^{*} \in S_{Z}, \Phi\left(x^{*}\right)$ is norm closed and

(1) $\Phi\left(x^{*}\right) \subset\left\{y^{*} \in Y^{*}:\left.y^{*}\right|_{x}=x^{*},\left\|y^{*}\right\| \leqq 1\right\}$.

Proof. Let us define $\Phi$ as follows: if $x^{*} \in S_{z}$ is not an extreme point of $S_{z}$ then $\Phi\left(x^{*}\right)$ is equal to the second member of (1) and if $x^{*}$ is an extreme point of $S_{Z}$ then $\Phi\left(x^{*}\right)=\left\{\psi\left(x^{*}\right)\right\}$. 
It is easily seen that $\Phi$ is affine, satisfies (1) and $\Phi\left(x^{*}\right)$ is closed for every $x^{*} \in S_{z}$. It remains to show that $\Phi$ is lower semicontinuous.

Let $U$ be an open subset of $Y^{*}$ and pick $x_{0}^{*} \in\left\{x^{*} \in S_{z}: \Phi\left(x^{*}\right) \cap U \neq \varnothing\right\}=M$. If $\left\|x_{0}^{*}\right\|<1$ then the argument used in the proof of Corollary 4.2 shows that $x_{0}^{*}$ is an interior point of $M$. If $\left\|x_{0}^{*}\right\|=1$ and $x_{0}^{*}$ is an extreme point of $S_{Z}$ then

$$
x_{0}^{*} \in S_{Z} \cap \psi^{-1}(U) \subset M
$$

and again, $x_{0}^{*}$ is an interior point of $M$.

Assume now that $1=\left\|x_{0}^{*}\right\|$ and $x_{0}^{*}$ is not an extreme point of $S_{z}$. Then there exist $x^{* *}, x^{\prime *} \in Z$ with $\left\|x^{* *}\right\|=\left\|x^{\prime *}\right\|=1, \frac{1}{2}\left(x^{*}+x^{\prime \prime *}\right)=x_{0}^{*}, x^{\prime *} \neq x_{0}^{*} \neq x^{\prime *}$. It is enough to prove that for any sequence $\left\{x_{n}^{*}\right\}_{n=1}^{\infty} \subset S_{Z}$ with $\left\|x_{n}^{*}-x_{0}^{*}\right\| \rightarrow 0$ almost all the terms belong to $M$ since from this results that $x_{0}$ is an interior point of $M$.

Starting from a certain $N$ all the terms of such a sequence belong to the triangle whose vertices are $0, x^{\prime *}, x^{\prime *}$ and no $x_{n}^{*}$ is an extreme point of $S_{Z}$. Hence

$$
x_{n}^{*}=\alpha_{n} x^{*}+\beta_{n} x^{\prime *}, \quad n \geqq N
$$

with $\alpha_{n} \geqq 0, \beta_{n} \geqq 0, \alpha_{n}+\beta_{n} \leqq 1$. Clearly

$$
\lim _{n \rightarrow \infty} \alpha_{n}=\lim _{n \rightarrow \infty} \beta_{n}=\frac{1}{2} \text {. }
$$

By the definition of $M, \Phi\left(x_{0}^{*}\right) \cap U \neq \varnothing$. Pick $y_{0}^{*} \in \Phi\left(x_{0}^{*}\right) \cap U$ and let $y^{*}, y^{\prime \prime}$ be norm preserving extensions to $Y$ of $x^{\prime *}$ respectively $x^{\prime \prime *}$. Define for $n \geqq N$

$$
\begin{aligned}
y_{n}^{*} & =\left(\alpha_{n}-\beta_{n}\right) y^{*}+2 \beta_{n} y_{0}^{*} & & \text { if } \alpha_{n} \geqq \beta_{n}, \\
& =\left(\beta_{n}-\alpha_{n}\right) y^{n *}+2 \alpha_{n} y_{0}^{*} & & \text { if } \alpha_{n}<\beta_{n} .
\end{aligned}
$$

Then $\left\|y_{n}^{*}\right\| \leqq 1$ and $\left.y_{n}^{*}\right|_{x}=x_{n}^{*}$ which means that $y_{n}^{*} \in \Phi\left(x_{n}^{*}\right)$. Moreover $\left\|y_{n}^{*}-y_{0}^{*}\right\| \rightarrow 0$ therefore $\Phi\left(x_{n}^{*}\right) \cap U \neq \varnothing$ if $n$ is sufficiently large. This concludes the proof of the lemma.

THEOREM 4.5. Let $X$ be a Banach space whose closed unit ball has at least one extreme point. The following statements are equivalent:

(i) There is a simplex $K$ such that $X$ is isometrically isomorphic with $A(K)$.

(ii) For any two Banach spaces $Y \subset Z$ such that there exists a C.N.P.E. map from $Y^{*}$ to $Z^{*}$, any operator $T: Y \rightarrow X$ with $\operatorname{dim} T(Y) \leqq 2$ has a compact norm preserving extension from $Z$ to $X$.

Proof. (i) $\Rightarrow$ (ii). We identify $X$ with $A(K)$. If $\operatorname{dim} T(Y)=1$ there is nothing to prove, so assume $\operatorname{dim} T(Y)=2$. Without loss of generality we can also assume that $\|T\|=1$.

Let $\chi$ be the function from $K$ to $S_{Y *}$ given by Lemma 4.1 and denote by $W$ the subspace of $Y^{*}$ spanned by $\chi(K)$. Clearly $\operatorname{dim} W=2$. Let $\Phi: S_{W} \rightarrow 2^{z^{*}}$ be the map given by Lemma 4.4. Then $\Phi \circ \chi$ satisfies the conditions of Theorem 3.1, and admits an affine continuous selection $\tilde{\chi}: K \rightarrow S_{Z^{*}}$. The operator $\tilde{T}: Z \rightarrow A(K)$ corresponding to $\tilde{\chi}$ is the desired one. 
(ii) $\Rightarrow$ (i). It is enough to suppose that (ii) is satisfied for $Y$ and $Z$ with $\operatorname{dim} Y=2$, $\operatorname{dim} Z=3$.

We shall show that if $Y \subset Z$ are Banach spaces with $\operatorname{dim} Y=2, \operatorname{dim} Z=3$, without any other restrictions on them, then any operator from $Y$ to $X$ has an almost norm preserving extension from $Z$ to $X$. By [15, Theorem 6.1 (16)] and [21] this implies (i).

Let $\varepsilon>0$ and let $P_{\varepsilon}$ be a centrally symmetric polyhedron in $Z$ such that $P_{\varepsilon} \subset S_{Z}$ $\subset(1+\varepsilon) P_{\varepsilon}$. Denote by \|\| the norm induced by $P_{\varepsilon}$ in $Z$ (and in $Y$ ). Then for any $z \in Z$

$$
\|z\| \leqq\|z\| \leqq(1+\varepsilon)\|z\| \text {. }
$$

If $T: Y \rightarrow X$ we have

$$
\|T\| \leqq\|T\| \leqq(1+\varepsilon)\|T\| .
$$

$P_{\varepsilon} \cap Y$ is a (two-dimensional) polyhedron and by [15, Theorem 7.3] there exists a C.N.P.E. map from $Y^{*}$ to $Z^{*}$ when $Y$ and $Z$ are equipped with the norm \|\|$\|$. By (ii) $T$ has an extension $\tilde{T}: Z \rightarrow X$ with $\|\tilde{T}\|=\|T\|$. If we come back to the initial norm of $Z$ then

$$
\|\tilde{T}\| \leqq(1+\varepsilon)\|\tilde{T}\|=(1+\varepsilon)\|T\| \leqq(1+\varepsilon)\|T\|
$$

which means that we found an almost norm preserving extension of $T$ and this concludes the proof of the theorem.

5. In this section we give two examples related to the results of $\$ 4$. The first one shows that the implication (i) $\Rightarrow$ (ii) of Theorem 4.5 cannot be strengthened adding the requirement $\operatorname{dim} \tilde{T}(Z)<\infty$ even if $X$ is the space of continuous functions over a compact Hausdorff space.

EXAMPLE 5.1. Let $p>1$ and not an even integer. In [14] it was proved that $l_{p}$ contains a two-dimensional subspace $Y$ such that the unique (by [19]) norm preserving extensions to $l_{p}$ of the functionals belonging to the boundary of $S_{Y^{*}}$ span an infinite-dimensional subspace of $l_{q}=l_{p}^{*}(1 / p+1 / q=1)$. On the other hand, by $[15, \mathrm{pp} .87-88]$, the norm preserving map from $Y^{*}$ to $l_{q}$ is norm continuous since $l_{q}$ is uniformly convex.

Denote by $T$ the natural embedding of $Y$ into $C\left(S_{\mathrm{Y}^{*}}\right)$. Clearly $\|T\|=1$ and $\operatorname{dim} T(Y)=2$. We claim that for any compact norm preserving extension $\tilde{T}$ of $T$ from $l_{p}$ to $C\left(S_{\mathrm{Y}^{*}}\right)$ we have $\operatorname{dim} \tilde{T}\left(l_{p}\right)=\infty$. Indeed, if $\tilde{T}$ is such an extension, it corresponds by $[8$, p. 490$]$ to a continuous function $\tilde{\chi}: S_{Y^{*}} \rightarrow l_{q}$ such that for every $y^{*} \in S_{Y^{*}}, \tilde{\chi}\left(y^{*}\right)$ is an extension of $y^{*}$ to $l_{p}$ and $\left\|\tilde{\chi}\left(y^{*}\right)\right\| \leqq 1$. From the mentioned property of $Y$ we infer that the image by $\tilde{\chi}$ of the boundary of $S_{Y^{*}}$ is not contained in any finite-dimensional subspace of $l_{q}$. Consequently

$$
\operatorname{dim} \tilde{T}\left(l_{q}\right)=\operatorname{dim} \tilde{T}^{*}\left(C^{*}\left(S_{Y^{*}}\right)\right)=\infty .
$$

Now we are going to exhibit two Banach spaces $X \subset Y$ with $\operatorname{dim} X=3, \operatorname{dim} Y=4$, a simplex $K$ and an operator $T: X \rightarrow A(K)$ without norm preserving extension 
from $Y$ to $A(K)$ although there is a C.N.P.E. map from $X^{*}$ to $Y^{*}$. For this we need a lemma whose proof is given in the Appendix.

If $0 \neq \xi=(\xi(1), \xi(2), \ldots) \in l_{1}$, then $R \xi$ denotes the subspace of $l_{1}$ generated by $\xi$, $S_{\xi}=\{i: \xi(i) \neq 0\}$ and for every $x \in l_{1}, P_{\xi}(x)$ is the set of the nearest points to $x$ from $R \xi$. Since $R \xi$ is locally compact, $P_{\xi}(x) \neq \varnothing$ for each $x \in l_{1}$.

LEMMA 5.2. There exists a continuous function $\lambda: l_{1} \rightarrow(-\infty, \infty)$ with $\lambda(x) \xi \in P_{\xi}(x)$ for every $x \in l_{1}$ if and only if there do not exist two disjoint sets $N_{1}, N_{2}$ of natural numbers such that:

(i) $N_{1} \cup N_{2}=S_{\xi}$;

(ii) $N_{1}, N_{2}$ are both infinite and

(iii) $\sum_{i \in N_{1}}|\xi(i)|=\sum_{i \in N_{2}}|\xi(i)|$.

Example 5.3. Let $A$ be the sequence space used for Example 3.2 and $K$ the corresponding simplex. Put

$$
\xi=\left(-\frac{1}{2},-\frac{1}{2}, \frac{1}{2}, \frac{1}{2^{2}}, \ldots, \frac{1}{2^{n}}, \ldots\right) \in l_{1} .
$$

It is easy to check that the conditions of Lemma 5.2 are fulfilled so there exists a continuous function $\lambda: l_{1} \rightarrow(-\infty, \infty)$ with $\lambda(x) \xi \in P_{\xi}(x)$ for every $x \in l_{1}$. Let $\left\{e_{n}\right\}_{n=1}^{\infty}$ be the unit vectors of $l_{1}$ and denote by $Z$ the subspace of $l_{1}$ spanned by $e_{1}, e_{2}$ and the vectors:

$\xi \in Z$ since

$$
\begin{aligned}
& \eta_{1}=\left(0,0, \frac{1}{2}, \frac{1}{2^{2}}, \ldots, \frac{1}{2^{n}}, \ldots\right) \\
& \eta_{2}=\left(0,0, \frac{1}{4}, \frac{1}{4} \cdot \frac{3}{4}, \ldots, \frac{1}{4} \cdot\left(\frac{3}{4}\right)^{n-1}, \ldots\right) .
\end{aligned}
$$

(1) $\xi=\eta_{1}-\left(e_{1}+e_{2}\right) / 2$.

Let $Y=Z^{*}$ and denote by $X$ the annihilator of $R \xi$ in $Y$. From the existence of a continuous approximation by nearest points and from [15, Lemma 7.4] we infer that there is a C.N.P.E. map from $X^{*}$ to $Y^{*}(=Z)$. Clearly $\operatorname{dim} X=3, \operatorname{dim} Y=4$.

Let

Then

$$
a_{n}=\frac{1}{2 c_{n}}\left(\frac{3}{2}\right)^{n-1}, \quad b_{n}=\frac{-1}{c_{n}}, \quad c_{n}=\left(\frac{3}{4}\right)^{n-1}\left(1+2^{n-2}\right)-1
$$

$$
c_{n}>0, \quad n=1,2, \ldots, \quad \lim _{n \rightarrow \infty} a_{n}=1, \quad \lim _{n \rightarrow \infty} b_{n}=0 .
$$

Consider the following sequence of $Z$ :

$$
z_{1}=e_{1}, \quad z_{2}=e_{2}, \quad z_{n}=a_{n} \eta_{1}+b_{n} \eta_{2}, \quad n=3,4, \ldots
$$

An easy calculation shows that $\left\|z_{n}\right\|=1, n=1,2, \ldots$

Define $T: X \rightarrow A$ by

$$
T(x)=\left\{z_{n}(x)\right\}_{n=1}^{\infty}, \quad x \in X .
$$


Since $\left\|z_{n}-\eta_{1}\right\| \rightarrow 0$ and (1) implies that

$$
\left.\eta_{1}\right|_{x}=\left.\frac{e_{1}+e_{2}}{2}\right|_{x}
$$

it is clear that $T(x) \in A$. We have

$$
\|T(x)\|=\sup _{n}\left|z_{n}(x)\right| \leqq\|x\| .
$$

Thus $\|T\| \leqq 1$. We shall prove that $\|T\|=1$. Let $\chi: K \rightarrow X^{*}(=Z / R \xi)$ be the affine continuous function corresponding to $T$ by Lemma 4.1. Obviously $\chi\left(k_{n}\right)=\phi\left(z_{n}\right)$ where $\phi$ is the canonic map $\phi: Z \rightarrow Z / R \xi$. It is enough to show that $\left\|\chi\left(k_{1}\right)\right\|$ $=\left\|\phi\left(z_{1}\right)\right\|=1$. We have

$$
\left\|\phi\left(z_{1}\right)\right\|=\inf _{\alpha}\left\|z_{1}+\alpha \xi\right\|
$$

and

$$
\left\|z_{1}+\alpha \xi\right\|=\frac{|2-\alpha|}{2}+\frac{|\alpha|}{2}+|\alpha| \sum_{i=1}^{\infty} \frac{1}{2^{i}}=\frac{3|\alpha|}{2}+\frac{|2-\alpha|}{2} .
$$

Thus $\left\|z_{1}+\alpha \xi\right\|=1 \Leftrightarrow \alpha=0$ and $\left\|\phi\left(z_{1}\right)\right\|=1$.

For a subsequent argument we need also to compute $\left\|\phi\left(z_{n}\right)\right\|, n=2,3, \ldots$ If $n=2$ then the same calculation as above shows that $\left\|z_{2}+\alpha \xi\right\|=1 \Leftrightarrow \alpha=0$ and $\left\|\phi\left(z_{2}\right)\right\|=1$.

Assume now $n \geqq 3$. Then

$$
\left\|z_{n}+\alpha \xi\right\|=|\alpha|+\sum_{n=1}^{\infty}\left|\frac{1}{c_{n}}\left[2^{n-1-2}\left(\frac{3}{4}\right)^{n-1}-2^{-2}\left(\frac{3}{4}\right)^{i-1}\right]+\frac{\alpha}{2^{i}}\right| .
$$

Let

Then

$$
\alpha_{i}^{n}=\frac{1}{2 c_{n}}\left[\left(\frac{3}{2}\right)^{i-1}-\left(\frac{3}{2}\right)^{n-1}\right]
$$

$$
\alpha \geqq \alpha_{i}^{n} \Leftrightarrow \frac{1}{c_{n}}\left[2^{n-i-2}\left(\frac{3}{4}\right)^{n-1}-2^{-2}\left(\frac{3}{4}\right)^{i-1}\right]+\frac{\alpha}{2^{i}} \geqq 0
$$

and $\alpha_{n}^{n}=0, \alpha_{i}^{n}<\alpha_{i+1}^{n}, \lim _{i \rightarrow \infty} \alpha_{i}^{n}=\infty$.

Let $\alpha<\alpha_{1}^{n}$. From (2) we have

$$
\left\|z_{n}+\alpha \xi\right\|=-2 \alpha-\frac{2^{n-2}(3 / 4)^{n-1}-1}{c_{n}}>-2 \alpha_{1}^{n}-\frac{2^{n-2}(3 / 4)^{n-1}-1}{c_{n}}=\frac{\frac{1}{2}(3 / 2)^{n-1}}{c_{n}}>1 .
$$

Let $\alpha_{i}^{n} \leqq \alpha<\alpha_{i+1}^{n} \leqq 0(1 \leqq i \leqq n-1)$. Then

$$
\begin{aligned}
\left\|z_{n}+\alpha \xi\right\| & =-\frac{\alpha}{2^{i-1}}+\frac{2^{n-2}(3 / 4)^{n-1}\left(1-1 / 2^{i-1}\right)+2(3 / 4)^{i}-1}{c_{n}} \\
& >-\frac{\alpha_{i+1}^{n}}{2^{i-1}}+\frac{2^{n-2}(3 / 4)^{n-1}\left(1-1 / 2^{i-1}\right)+2(3 / 4)^{i}-1}{c_{n}} \\
& =\frac{(3 / 4)^{i}+\frac{1}{2}(3 / 2)^{n-1}-1}{c_{n}} \geqq 1
\end{aligned}
$$


Let $0 \leqq \alpha_{i}^{n}<\alpha \leqq \alpha_{i+1}^{n}(i \geqq n)$. Then

$$
\begin{aligned}
\left\|z_{n}+\alpha \xi\right\| & =\alpha\left(2-\frac{1}{2^{i-1}}\right)+\frac{2^{n-2}(3 / 4)^{n-1}\left(1-1 / 2^{i-1}\right)+2(3 / 4)^{i}-1}{c_{n}} \\
& >\alpha_{i}^{n}\left(2-\frac{1}{2^{i-1}}\right)+\frac{2^{n-2}(3 / 4)^{n-1}\left(1-1 / 2^{i-1}\right)+2(3 / 4)^{i}-1}{c_{n}} \\
& =\frac{(3 / 2)^{i-1}+(3 / 4)^{i-1}-\frac{1}{2}(3 / 2)^{n-1}-1}{\frac{1}{2}(3 / 2)^{n-1}+(3 / 4)^{n-1}-1} .
\end{aligned}
$$

Since $(3 / 2)^{t}+(3 / 4)^{t}$ is an increasing function for $t \geqq 0$, we have

$$
(3 / 2)^{i-1}+(3 / 4)^{i-1} \geqq(3 / 2)^{n-1}+(3 / 4)^{n-1}
$$

and consequently $\left\|z_{n}+\alpha \xi\right\|>1$.

Summarizing the results, we found that

(3) $\left\|z_{n}+\alpha \xi\right\|=1 \Leftrightarrow \alpha=0$ and $\left\|\phi\left(z_{n}\right)\right\|=1, n=1,2, \ldots$

Suppose now that $T$ has an extension $\tilde{T}: Y \rightarrow A$ with $\|\tilde{T}\|=1$. Then there is an affine continuous function $\tilde{\chi}: K \rightarrow S_{Y^{*}}=S_{Z}$ such that $\left\|\tilde{\chi}\left(k_{n}\right)\right\|=1$ and $\tilde{\chi}\left(k_{n}\right) \in z_{n}+R \xi$, $n=1,2, \ldots$ From (3) we infer $\tilde{\chi}\left(k_{n}\right)=z_{n}, n=1,2, \ldots$ Hence

$$
\tilde{\chi}\left(k_{1}+k_{2}\right) / 2=\left(z_{1}+z_{2}\right) / 2 \text {. }
$$

On the other hand,

$$
\tilde{\chi}\left(k_{1}+k_{2}\right) / 2=\lim _{n \rightarrow \infty} \tilde{\chi}\left(k_{n}\right)=\lim _{n \rightarrow \infty} z_{n}=\eta_{1}
$$

and $\eta_{1} \neq\left(z_{1}+z_{2}\right) / 2$. This contradiction shows that such an extension of $T$ cannot exist.

REMARK. We can choose $Y=c_{0}$ and $X$ to be the annihilator of $R \xi$ in $c_{0}$. Defining $T: X \rightarrow A$ as above by $T(x)=\left\{z_{n}(x)\right\}$ we get a compact operator without norm preserving extension from $Y$ to $A$. For these $X$ and $Y$ there exists a C.N.P.E. map from $X^{*}$ to $Y^{*}$ and $X$ (as well as $Y$ ) is a polyhedral space [11]. Lindenstrauss proved [15, Theorem 7.9(a)] that an operator from a finite-dimensional polyhedral space into a space with the F.2.I.P. has always a compact norm preserving extension. The above modified example shows that this theorem cannot be generalized for infinite-dimensional polyhedral spaces even in the presence of a C.N.P.E. map between the adjoint spaces.

APPENDIX. We are going now to prove Lemma 5.2. We shall use the following particular case of a theorem of Kripke and Rivlin [12, Theorem 2]:

LEMMA. Let $x \in l_{1}$. Then $\lambda_{0} \xi \in P_{\xi}(x)$ if and only if

$$
\left|\sum_{x(i) / \xi(i)>\lambda_{0}}\right| \xi(i)\left|-\sum_{x(i) / \xi(i)<\lambda_{0}}\right| \xi(i)|| \leqq \sum_{x(i) / \xi(i)=\lambda_{0}}|\xi(i)|
$$

(everywhere $\left.i \in S_{\xi}\right)$. If (1) holds with $<$ then $P_{\xi}(x)=\left\{\lambda_{0} \xi\right\}$. 
Proof of Lemma 5.2. Suppose that there exist two infinite sets of natural numbers $N_{1}=\left\{i_{1}, i_{2}, \ldots, i_{k}, \ldots\right\}, N_{2}=\left\{j_{1}, j_{2}, \ldots, j_{k}, \ldots\right\}\left(i_{k}<i_{k+1}, j_{k}<j_{k+1}\right)$ such that $N_{1} \cap N_{2}=\varnothing, N_{1} \cup N_{2}=S_{\xi}$ and

$$
\sum_{i \in N_{1}}|\xi(i)|=\sum_{i \in N_{2}}|\xi(i)| \text {. }
$$

We shall show that there is no real continuous function $\lambda$ defined on $l_{1}$ such that $\lambda(x) \xi \in P_{\xi}(x)$ for every $x \in l_{1}$.

Let $x_{n}^{\prime}, x_{n}^{n} \in l_{1}, n=1,2, \ldots$ be the following sequences:

$$
\begin{aligned}
x_{n}^{\prime}(i) & =\xi\left(i_{k}\right), & & i=i_{k}, \\
& =\xi\left(j_{n}\right), & & i=j_{n}, \\
& =-\xi\left(j_{k}\right), & & i=j_{k}, k \neq n, \\
& =0, & & i \notin N_{1} \cup N_{2}, \\
x_{n}^{\prime \prime}(i) & =\xi\left(i_{k}\right), & & i=i_{k}, k \neq n, \\
& =-\xi\left(i_{n}\right), & & i=i_{n}, \\
& =-\xi\left(j_{k}\right), & & i=j_{k}, \\
& =0, & & i \notin N_{1} \cup N_{2} .
\end{aligned}
$$

Using the above lemma it is easy to see that $P_{\xi}\left(x_{n}^{\prime}\right)=\{\xi\}, P_{\xi}\left(x_{n}^{\prime \prime}\right)=\{-\xi\}, n=1,2, \ldots$ Obviously $\left\{x_{n}^{\prime}\right\}_{n=1}^{\infty},\left\{x_{n}^{\prime \prime}\right\}_{n=1}^{\infty}$ converge in the norm of $l_{1}$ to $x_{0}$ where

$$
\begin{aligned}
x_{0}(i) & =\xi(i), & & i \in N_{1}, \\
& =-\xi(i), & & i \in N_{2}, \\
& =0, & & i \notin N_{1} \cup N_{2} .
\end{aligned}
$$

Hence, no function $\lambda: l_{1} \rightarrow(-\infty, \infty)$ with $\lambda(x) \xi \in P_{\xi}(x)$ for every $x \in l_{1}$ can be continuous at $x_{0}$. This concludes the "only if" part of the lemma.

Assume now that there are no such sets $N_{1}, N_{2}$. We distinguish two cases: (1) $S_{\xi}$ is infinite; (2) $S_{\xi}$ is finite.

(1) The first step is to define the function $\lambda: l_{1} \rightarrow(-\infty, \infty)$ such that $\lambda(x) \xi \in P_{\xi}(x)$ for each $x \in l_{1}$; afterwards we shall show that it is continuous.

If for a certain $x \in l_{1}$ there is a real $\lambda_{0}$ such that (1) holds with $<$ then, of course, $\lambda(x)=\lambda_{0}$. Clearly $\lambda(x) \xi \in P_{\xi}(x)$.

Fix now $x \in l_{1}$ and suppose that there is a real $\lambda_{c}$ such that (1) holds with equality sign. Denote $S=S_{\xi}$ and

$$
B^{\prime}=\left\{i \in S: x(i) / \xi(i) \geqq \lambda_{0}\right\}, \quad C^{\prime}=\left\{i \in S: x(i) / \xi(i) \leqq \lambda_{0}\right\} .
$$

One of the following equalities is valid:

$$
\begin{aligned}
\sum_{i \in B^{\prime}}|\xi(i)| & =\sum_{i \in S \sim B^{\prime}}|\xi(i)|, \\
\sum_{i \in C^{\prime}}|\xi(i)| & =\sum_{i \in S \sim C^{\prime}}|\xi(i)| .
\end{aligned}
$$


If (2) is valid, by our hypothesis, one and only one of the sets $B^{\prime}, S \sim B^{\prime}$ is finite. If $B^{\prime}$ is finite define

$$
\lambda(x)=\min _{i \in B^{\prime}} \frac{x(i)}{\xi(i)}
$$

and if $S \sim B^{\prime}$ is finite let

$$
\lambda(x)=\max _{i \in S \sim B^{\prime}} \frac{x(i)}{\xi(i)}
$$

Similarly, if (3) is true, one and only one of the sets $C^{\prime}, S \sim C^{\prime}$ is finite. We define

$$
\lambda(x)=\max _{i \in C^{\prime}} \frac{x(i)}{\xi(i)}
$$

if $C^{\prime}$ is finite and

$$
\lambda(x)=\min _{i \in S \sim C^{\prime}} \frac{x(i)}{\xi(i)}
$$

if $S \sim C^{\prime}$ is finite. If both (2) and (3) are valid (then $\left\{i \in S: x(i) / \xi(i)=\lambda_{0}\right\}=\varnothing$ ) there is no contradiction in the definition of $\lambda(x)$.

Let

$$
\begin{gathered}
A_{x}=\{i \in S: x(i) / \xi(i)=\lambda(x)\}, \quad B_{x}=\{i \in S: x(i) / \xi(i)>\lambda(x)\}, \\
C_{x}=\{i \in S: x(i) / \xi(i)<\lambda(x)\} .
\end{gathered}
$$

Then always $A_{x} \neq \varnothing$ and if $\lambda(x)$ is defined by one of the relations (4)-(7) we have

$$
\left|\sum_{i \in B_{x}}\right| \xi(i)\left|-\sum_{i \in C_{x}}\right| \xi(i)||=\sum_{i \in A_{x}}|\xi(i)| .
$$

This implies that $\lambda(x) \xi \in P_{\xi}(x)$. Moreover, in this case, if

$$
\sum_{i \in B_{x}}|\xi(i)|-\sum_{i \in C_{x}}|\xi(i)|=\sum_{i \in A_{x}}|\xi(i)|
$$

then $A_{x} \cup C_{x}$ is finite and conversely; if

$$
\sum_{i \in C_{\boldsymbol{x}}}|\xi(i)|-\sum_{i \in B_{\boldsymbol{x}}}|\xi(i)|=\sum_{i \in A_{\boldsymbol{x}}}|\xi(i)|
$$

then $A_{x} \cup B_{x}$ is finite and conversely.

Now we can show that $\lambda$ is continuous. Fix $x \in l_{1}$ and assume first that $P_{\xi}(x)$ $=\{\lambda(x) \xi\}$. Let $\left\{u_{n}\right\}_{n=1}^{\infty} \subset l_{1}$ be a sequence convergent to $x$. Then

$$
\begin{aligned}
\|x-\lambda(x) \xi\| & \leqq\left\|x-\lambda\left(u_{n}\right) \xi\right\| \leqq\left\|x-u_{n}\right\|+\left\|u_{n}-\lambda\left(u_{n}\right) \xi\right\| \\
& =\left\|x-u_{n}\right\|+\inf _{\alpha}\left\|u_{n}-\alpha \xi\right\| \leqq 2\left\|x-u_{n}\right\|+\inf _{\alpha}\|x-\alpha \xi\| \\
& =2\left\|x-u_{n}\right\|+\|x-\lambda(x) \xi\| .
\end{aligned}
$$

From this we infer that $\left\{\lambda\left(u_{n}\right)\right\}_{n=1}^{\infty}$ is a bounded sequence and if $\lambda\left(u_{n_{k}}\right) \rightarrow \alpha$ then $\|x-\lambda(x) \xi\|=\|x-\alpha \xi\|$ which, by our assumption about $P_{\xi}(x)$, implies that $\alpha=\lambda(x)$. This proves the continuity of $\lambda$ at $x$. 
Assume now that $P_{\xi}(x)$ contains more than one point. Then $\lambda(x)$ is defined by one of the relations (4)-(7). Hence (8) or (9) holds. We shall carry out the proof for the case that (8) holds, the proof for the remaining case being similar.

Let $\varepsilon>0$ and put

$$
V=\left\{u \in l_{1}:|u(i)-x(i)|<|\xi(i)| \varepsilon, i \in A_{x} \cup C_{x}\right\} .
$$

$A_{x} \cup C_{x}$ is finite therefore $V$ is an open neighborhood of $x$. We claim that $u \in V$ $\Rightarrow \lambda(u)<\lambda(x)+\varepsilon$. Suppose this were false. Then there is $u^{\prime} \in V$ with $\lambda\left(u^{\prime}\right) \geqq \lambda(x)+\varepsilon$. If $i \in A_{x} \cup C_{x}$ we have

$$
u^{\prime}(i) / \xi(i)<x(i) / \xi(i)+\varepsilon \leqq \lambda(x)+\varepsilon \leqq \lambda\left(u^{\prime}\right)
$$

which yields

$$
A_{x} \cup C_{x} \subset C_{u^{\prime}}, \quad B_{x} \supset A_{u^{\prime}} \cup B_{u^{\prime}}
$$

From

$$
\left|\sum_{i \in B_{u^{\prime}}}\right| \xi(i)\left|-\sum_{i \in C_{u^{\prime}}}\right| \xi(i)|| \leqq \sum_{i \in A_{u^{\prime}}}|\xi(i)|
$$

and from (8) and (11) we infer that

$$
\sum_{i \in B_{x}}|\xi(i)|=\sum_{i \in A_{x} \cup C_{x}}|\xi(i)| \leqq \sum_{i \in C_{u^{\prime}}}|\xi(i)| \leqq \sum_{i \in A_{u^{\prime}} \cup B_{u^{\prime}}}|\xi(i)| .
$$

This together with (11) implies that $B_{x}=A_{u^{\prime}} \cup B_{u^{\prime}}$ and

$$
\sum_{i \in C_{u^{\prime}}}|\xi(i)|-\sum_{i \in B_{u^{\prime}}}|\xi(i)|=\sum_{i \in A_{u^{\prime}}}|\xi(i)| .
$$

Hence $\lambda\left(u^{\prime}\right)$ is defined by one of the relations (4)-(7) and for $u^{\prime},(9)$ holds. But this means that $A_{u^{\prime}} \cup B_{u^{\prime}}=B_{x}$ is finite which contradicts the assumption that $A_{x} \cup C_{x}$ is finite. Our assertion about $V$ is proved.

On the other hand, there is a neighborhood $U$ of $x$ such that $u \in U \Rightarrow \lambda(u)$ $>\lambda(x)-\varepsilon$. If this were false there would exist a sequence $\left\{u_{n}\right\}_{n=1}^{\infty} \subset l_{1}$ with $\left\|u_{n}-\dot{x}\right\|$ $\rightarrow 0$ and $\lambda\left(u_{n}\right) \leqq \lambda(x)-\varepsilon$. By $(10),\left\{\lambda\left(u_{n}\right)\right\}_{n=1}^{\infty}$ is a bounded sequence and if $\lambda\left(u_{n_{k}}\right) \rightarrow \alpha$ then $\alpha \xi \in P_{\xi}(x)$. Since $\alpha \leqq \lambda(x)-\varepsilon$ we have

Hence

$$
\sum_{x(i) / \xi(i) \leqq \alpha}|\xi(i)|<\sum_{i \in A_{x} \cup C_{x}}|\xi(i)|=\sum_{i \in B_{x}}|\xi(i)|<\sum_{x(i) / \xi(i)>\alpha}|\xi(i)| .
$$

$$
\sum_{x(i) / \xi(i)>\alpha}|\xi(i)|-\sum_{x(i) / \xi(i)<\alpha}|\xi(i)|>\sum_{x(i) / \xi(i)=\alpha}|\xi(i)|
$$

from which we infer that $\alpha \xi \notin P_{\xi}(x)$. This is a contradiction; thus there is a neighborhood of $x$ with the desired property.

Now, $u \in U \cap V \Rightarrow|\lambda(x)-\lambda(u)|<\varepsilon$ and this means that $\lambda$ is continuous at $x$.

(2) $S_{\xi}$ is finite. Denote as usual by $\left\{e_{i}\right\}_{i=1}^{\infty}$ the unit vectors of $l_{1}$ and let $E$ be the subspace of $l_{1}$ spanned by $\left\{e_{i}: i \in S_{\xi}\right\}$. Clearly $R \xi \subset E$. The unit ball of $E$ is a polyhedron hence the polar body is also a polyhedron. The annihilator of $R \xi$ in $E^{*}$ is a 
finite-dimensional space having a polyhedron as unit ball. By [15, Theorem 7.3, Lemma 7.4] there is a continuous function $\lambda: E \rightarrow(-\infty, \infty)$ such that $\lambda(x) \xi \in P_{\xi}(x)$ for every $x \in E$.

Let $T$ be the canonical projection of $l_{1}$ onto $E$. For any $x \in l_{1}$ we have

$$
\begin{aligned}
\|x-\lambda(T(x)) \xi\| & =\|T(x)-\lambda(T(x)) \xi\|+\sum_{i \notin S_{\xi}}|x(i)| \\
& \leqq\|T(x)-\alpha \xi\|+\sum_{i \notin S_{\xi}}|x(i)|=\|x-\alpha \xi\| .
\end{aligned}
$$

Hence $\lambda(T(x)) \xi \in P_{\xi}(x)$ for every $x \in l_{1}$ and obviously $\lambda \circ T$ is a continuous function. This concludes the proof of the lemma.

REMARKS. One could give a direct proof in the second case too. For instance, one could show that the functions

$$
\lambda_{1}(x)=\inf \left\{\lambda: \lambda \xi \in P_{\xi}(x)\right\}, \quad \lambda_{2}(x)=\sup \left\{\lambda: \lambda \xi \in P_{\xi}(x)\right\}
$$

are continuous $\left(P_{\xi}(x)\right.$ is closed so $\left.\lambda_{i}(x) \xi \in P_{\xi}(x)\right)$.

The difference between the patterns we used in proving the two cases can be explained, perhaps, by the following observations. If $S_{\xi}$ is infinite the subset of $l_{1}$ consisting of the points for which there exists a real $\lambda_{0}$ such that (1) holds with $<$ is dense in $l_{1}$ therefore $\lambda$ is uniquely determined by its properties. If $S_{\xi}$ finite and $R \xi$ is not a Haar subspace of $l_{1}$, the subset of $l_{1}$ of all the points for which (1) holds with equality sign is nonvoid and open. Obviously, in this case any convex combination of $\lambda_{1}$ and $\lambda_{2}$ fulfils the requirements.

If $\xi$ satisfies the conditions of Lemma 5.2, $X$ is a Banach space such that $X^{*}=l_{1}$ and $Y$ is the annihilator of $R \xi$ in $X$ then, by [15, Lemma 7.4] there exists a C.N.P.E. map from $Y^{*}$ to $X^{*}$. Moreover, if $Z$ is any Banach space with $Z \supset Y$ there is a C.N.P.E. map from $Y^{*}$ to $Z^{*}$. This can be proved by the same argument used in the proof of Theorem 7.3(a) of [15]. This theorem states that if the adjoint of a Banach space $W$ is an $L$ space then there is a C.N.P.E. map from $W^{*}$ to the adjoint of every Banach space in which $W$ is contained. The space $Y$ enjoys this property but one can choose $\xi$ such that $Y^{*}$ is not an $L$ space.

\section{REFERENCES}

1. E. M. Alfsen, On the geometry of Choquet simplexes, Math. Scand. 15 (1964), 97-110.

2. - Boundary values for homomorphisms of compact convex sets, Math. Scand. 19 (1966), 113-121.

3. R. Arens, Extensions of functions on fully normal spaces, Pacific J. Math. 2 (1952), 11-22.

4. K. Borsuk, Über Isomorphie der Funktionalräume, Bull. Int. Acad. Polon. Sci. (1933), 1-10.

5. G. Choquet and P. A. Meyer, Existence et unicité des représentations intégrales dans les convexes compacts quelconques, Ann. Inst. Fourier (Grenoble) 13 (1963), 139-154.

6. M. M. Day, Normed linear spaces, Springer-Verlag, Berlin, 1962.

7. J. Dugundji, An extension of Tietze's theorem, Pacific J. Math. 1 (1951), 353-367.

8. N. Dunford and J. T. Schwartz, Linear operators, Part I, Interscience, New York, 1958.

9. D. A. Edwards, Minimum-stable wedges of semi-continuous functions, Math. Scand. 19 (1966), 15-26. 
10. E. G. Effros, Structure in simplexes, Matematisk Institut, Aarhus Universitet, 31 pp.

11. V. Klee, Polyhedral sections of convex bodies, Acta. Math. 103 (1960), 243-267.

12. B. R. Kripke and T. J. Rivlin, Approximation in the metric of $L^{1}(X, \mu)$, Trans. Amer. Math. Soc. 119 (1965), 101-122.

13. A. J. Lazar, On affine continuous functions on simplexes, dittoed notes, The Hebrew University, Jerusalem, $20 \mathrm{pp}$.

14. A. J. Lazar and M. Zippin, On finite-dimensional subspaces of Banach spaces, Israel J. Math. 3 (1965), 147-156.

15. J. Lindenstrauss, Extensions of compact operators, Mem. Amer. Math. Soc. No. 48 (1964), $112 \mathrm{pp}$.

16. —, A selection theorem, Israel J. Math. 2 (1964), 201-204.

17. E. A. Michael, Continuous selections. I, Ann. of Math. 63 (1956), 361-382.

18. - Selected selection theorems, Amer. Math. Monthly 63 (1956), 233-238.

19. R. R. Phelps, Uniqueness of Hahn-Banach extensions and unique best approximations, Trans. Amer. Math. Soc. 95 (1960), 238-255.

20. - Lectures on Choquet's Theorem, Van Nostrand, New York, 1966.

21. Z. Semadeni, Free compact convex sets, Bull. Acad. Polon. Sci. Sér. Sci. Math. Astronom. Phys. 13 (1964), 141-146.

The Hebrew University, JERUSALEM, ISRAEL 